\title{
An exponential formula for one-parameter semi-groups of nonlinear transformations
}

\author{
By J. W. NEUBERGER
}

(Received Oct. 26, 1965)

For a complete normed linear space $S$ consider a function $T$ from $[0, \infty)$ to the set of continuous transformations from $S$ to $S$ which satisfies:

(1) $T(x) T(y)=T(x+y)$ if $x, y>0$,

(2) $\|T(x) p-T(x) q\| \leqq\|p-q\|$ if $x \geqq 0, p, q$ are in $S$,

(3) if $p$ is in $S$ and $g_{p}(x)=T(x) p$ for all $x$ in $[0, \infty)$ then $g_{p}$ is continuous and $\lim _{x \rightarrow 0+} g_{p}(x)=p$.

If it is also specified that $T(x)$ is linear for all $x \geqq 0$, then one has a semigroup about which the following is known ([1] chapters 10, 11 and [3] sections 142, 143):

For all $p$ in some dense subset of $S, g_{p}^{\prime}(0)$ exists and if $A p=g_{p}^{\prime}(0)$ for all $p$ for which $g_{p}^{\prime}(0)$ exists, then $(I-x A)^{-1}$ exists, has domain $S$ and is continuous for all $x \geqq 0$. Moreover, if $p$ is in $S$ and $x \geqq 0$,

$$
\lim _{n \rightarrow \infty}\left\|(I-(x / n) A)^{-n} p-T(x) p\right\|=0 .
$$

It is the purpose of this note to add to assumptions (1)-(3) a differentiability condition (which, it turns out, holds in the linear special case) which implies an "exponential formula" suggested by (*). The results of this note give a nonlinear version of the linear strong case of [1] (section 11.5); previous work [2] (section 3) of this author gave a nonlinear version of the linear uniform case of [1] (section 11.2).

The differentiability condition mentioned above is:

(4) there is a dense subset $D$ of $S$ such that if $p$ is in $D$, then $g_{p}^{\prime}$ is continuous with domain $[0, \infty)$.

If $\delta>0$, denote $(1 / \delta)[T(\delta)-I]$ by $A_{\delta}$. The main result of this note follows.

Theorem. If (1)-(4) hold, $p$ is in $S$ and $x \geqq 0$, then

$$
\lim _{n \rightarrow \infty} \limsup _{\delta \rightarrow 0+}\left\|\left(I-(x / n) A_{\delta}\right)^{-n} p-T(x) p\right\|=0 .
$$

Consider first some lemmas.

LEMMA 1. Under conditions (1)-(3), if $\delta, x>0$, then $\left(I-x A_{\delta}\right)^{-1}$ exists and has domain $S$. 
Proof. Suppose $w$ is in $S$. A unique point $y$ of $S$ is sought so that $\left(I-x A_{\delta}\right) y=w$, that is, $y-(x / \delta) T(\delta) y+(x / \delta) y=w$, that is, $y=[\delta /(\delta+x)] w$ $+[x /(\delta+x)] T(\delta) y$. Define $K z=[\delta /(\delta+x)] w+[x /(\delta+x)] T(\delta) z$ for all $z$ in $S$. It is easily seen that $K$ is a contraction mapping. Hence there is a unique $y$ in $S$ so that $y=K y$. This proves the lemma.

LEMMA 2. Under conditions (1)-(3), if $\delta, x>0$, then

$$
\left\|\left(I-x A_{\delta}\right)^{-1} u-\left(I-x A_{\delta}\right)^{-1} v\right\| \leqq\|u-v\|
$$

for all $u, v$ in $S$.

Proof. Suppose that $\left(I-x A_{\delta}\right)^{-1} u=y$ and $\left(I-x A_{\delta}\right)^{-1} v=z z$. Then $y=[\delta /$ $(\delta+x)] u+[x /(\delta+x)] T(\delta) y$ and $z=[\delta /(\delta+x)] v+[x /(\delta+x)] T(\delta) z$ and hence, $\|y-z\|$ $\leqq[\delta /(\delta+x)]\|u-v\|+[x /(\delta+x)]\|T(\delta) y-T(\delta) z\| \leqq[\delta /(\delta+x)]\|u-v\|+[x /(\delta+x)]$ $\|y-z\|$. But this gives that $\|y-z\| \leqq\|u-v\|$ and hence the lemma is esta. blished.

LEMMA 3. Under conditions (1)-(3), if $\delta, x>0$, then $\left\|\left(I-x A_{\delta}\right)^{-1} p-T(x) p\right\|$ $\leqq x\left\|A_{x} p-A_{\delta} T(x) p\right\|$ for each $p$ in $S$.

ProOF.

$$
\begin{aligned}
& \left\|\left(I-x A_{\delta}\right)^{-1} p-T(x) p\right\| \leqq\left\|p-\left(I-x A_{\delta}\right) T(x) p\right\| \\
& \quad=\left\|[T(x)-I] p-x A_{\delta} T(x) p\right\|=x\left\|A_{x} p-A_{\delta} T(x) p\right\| .
\end{aligned}
$$

LEMMA 4. Suppose that each of $L$ and $M$ is a continuous transformation from $S$ to $S$ such that if $u$ and $v$ are in $S,\|L u-L v\| \leqq\|u-v\|$. Then for each positive integer $n,\left\|L^{n} p-M^{n} p\right\| \leqq \sum_{i=1}^{n}\left\|L M^{i-1} p-M^{i} p\right\|$ for all $p$ in $S$.

ProOF.

$$
\begin{aligned}
& \left\|L^{n} p-M^{n} p\right\|=\left\|\sum_{i=1}^{n}\left(L^{n-i+1} M^{i-1} p-L^{n-i} M^{i} p\right)\right\| \\
& \leqq \sum_{i=1}^{n}\left\|L^{n-i+1} M^{i-1} p-L^{n-i} M^{i} p\right\| \leqq \sum_{i=1}^{n}\left\|L M^{i-1} p-M^{i} p\right\| .
\end{aligned}
$$

Lemma 5. Under condition (4), suppose that $p$ is in $D, R$ is a bounded subinterval of $[0, \infty)$ and $\varepsilon>0$. There is $a \delta>0$ such that if $x, y$ are in $R$ and $0<|x-y|<\delta$, then $\max _{w \text { in }[x, y]}\left\|(x-y)^{-1}\left[g_{p}(x)-g_{p}(y)\right]-g_{p}^{\prime}(w)\right\|<\varepsilon$. hence

Proof. For $x$ and $y$ in $R, x \neq y$ and $w$ in $[x, y], g_{p}(x)-g_{p}(y)=\int_{y}^{x} g_{p}^{\prime}$ and

$$
\begin{aligned}
\left\|(x-y)^{-1}\left[g_{p}(x)-g_{p}(y)\right]-g_{p}^{\prime}(w)\right\|=\|(x-y)^{-1} \\
\quad \int_{y}^{x}\left(g_{p}^{\prime}-g_{p}^{\prime}(w)\right)\left\|\leqq \max _{c \text { in }[x, y]}\right\| g_{p}^{\prime}(c)-g_{p}^{\prime}(w) \| .
\end{aligned}
$$

The uniform continuity of $g_{p}^{\prime}$ on bounded intervals then gives the lemma.

PROOF OF THE THEOREM. The conclusion is obvious if $x=0$. Suppose $x>0$. Assume first that $r$ is in $D$. If $\delta>0$ and $n$ is a positive integer, then 


$$
\begin{aligned}
& \left\|\left(I-(x / n) A_{\delta}\right)^{-n} r-T(x) r\right\|=\left\|\left[\left(I-(x / n) A_{\delta}\right)^{-1}\right]^{n} r-[T(x / n)]^{n} r\right\| \\
& \leqq \sum_{i=1}^{n}\left\|\left(I-(x / n) A_{\delta}\right)^{-1} T(x(i-1) / n) r-T(x / n) T(x(i-1) / n) r\right\| \\
& \leqq \sum_{i=1}^{n}(x / n)\left\|A_{x / n} T(x(i-1) / n) r-A_{\delta} T(x i / n) r\right\| \\
& =(x / n) \sum_{i=1}^{n}\left\|(n / x)\left[g_{r}(x i / n)-g_{r}(x(i-1) / n)\right]-(1 / \delta)\left[g_{r}(\delta+x i / n)-g_{r}(x i / n)\right]\right\| \\
& \leqq(x / n)\left\{\sum_{i=1}^{n}\left\|(n / x)\left[g_{r}(x i / n)-g_{r}(x(i-1) / n)\right]-g_{r}^{\prime}(x i / n)\right\|\right. \\
& \left.\quad+\sum_{i=1}^{n}\left\|(1 / \delta)\left[g_{r}(\delta+i x / n)-g_{r}(x i / n)\right]-g_{r}^{\prime}(x i / n)\right\|\right\} .
\end{aligned}
$$

Suppose now, in addition, that $\varepsilon>0$. Denote by $\delta^{\prime}$ a positive number less than 1 so that if $0 \leqq v, u \leqq x+1$ and $0<|u-v|<\delta^{\prime}$, then $\max _{w \text { in }[u, v]} \|(u-v)^{-1}$ $\left[g_{r}(u)-g_{r}(v)\right]-g_{r}^{\prime}(w) \|<\varepsilon /(4 x)$. Denote by $N$ an integer so that $x / N<\delta^{\prime}$. If $n$ is an integer greater than $N$ and $0<\delta<\delta^{\prime},\left\|\left(I-(x / n) A_{\delta}\right)^{-n} r-T(x) r\right\|$ $\leqq(x / n) \sum_{i=1}^{n}(2 \varepsilon) /(4 x)=\varepsilon / 2$. From this it follows that $\limsup _{\delta \rightarrow 0+} \|\left(I-(x / n) A_{\delta}\right)^{-n} r$ $-T(x) r \|<\varepsilon$.

Suppose that $p$ is in $S$ and $\varepsilon>0$. Since $D$ is dense in $S$, there is a point $r$ of $D$ such that $\|p-r\|<\varepsilon / 6$. By the above argument, there is an integer $N$ and a $\delta^{\prime}>0$ such that if $n$ is an integer greater than $N$ and $0<\delta<\delta^{\prime}$, $\left\|\left(I-(x / n) A_{\delta}\right)^{-n} r-T(x) r\right\|<\varepsilon / 6$. For $\delta$ and $n$ chosen in such a way, $\| T(x) r$ $-T(x) p \|<\varepsilon / 6$ and $\left\|\left(I-(x / n) A_{\delta}\right)^{-n} r-\left(I-(x / n) A_{\delta}\right)^{-n} p\right\|<\varepsilon / 6$ (by repeated application of Lemma 2) which gives $\left\|\left(I-(x / n) A_{\delta}\right)^{-n} p-T(x) p\right\|<\varepsilon / 2$. From this it follows that $\lim _{\delta \rightarrow 0+} \sup \left\|\left(I-(x / n) A_{\delta}\right)^{-n} p-T(x) p\right\|<\varepsilon$ for all integers $n$ greater than $N$. This proves the theorem.

In closing it is noted that conditions (1)-(3) do not imply (4). This can be seen by considering the case in which $S$ is $E_{1}$ and

$$
T(x) p=\left\{\begin{array}{l}
p-x \text { if } p \geqq 1 \text { and } p-x \geqq 1, x \geqq 0 \\
1 \text { if } p \geqq 1 \text { and } p-x<1, x \geqq 0 \\
p \text { if } p<1, x \geqq 0 .
\end{array}\right.
$$

This author considers it likely that conditions (1)-(3) do imply interesting differentiability conditions and that the conclusion to the theorem (or perhaps a stronger conclusion) can be obtained using only conditions (1)-(3) together, perhaps, with some condition much weaker than (4). Investigations into these matters may well lead to a theory of semi-groups of nonlinear transformations which parallels rather completely the well developed linear case.

Emory University 


\section{References}

[1] E. Hille and R.S. Phillips, Functional analysis and semi-groups, rev. ed., Amer. Math. Soc. Colloquium Publications, vol. XXXI, 1957.

[2] J.W. Neuberger, A generator for a set of functions, [llinois J. Math., 9 (1965), 31-39.

[3] F. Riesz and B. Sz.-Nagy, Functional analysis, Ungar, New York, 1955. 